\title{
Control of Guide Disk Speed of Rotary piercer
}

\author{
Xiaoli Pan ${ }^{1,2}$ Dong Xiao ${ }^{1}$ Yong Yuan $^{1}$ Zhizhong Mao $^{1}$ Yuqing Chang $^{1}$ Fuli Wang $^{1}$ \\ ${ }^{1}$ Key Laboratory of Process Industry Automation of Ministry of Education and Liaoning Province, Northeast \\ University, Shenyang 110004, P. R. China \\ ${ }^{2}$ BaoSteel Corporation, Shanghai 201900, P. R. China
}

\begin{abstract}
It has been a long harassment to seamless tubes production that its productivity will be largely affected by the pause caused in adjusting the craft parameters of piercer. A rebuilding method of guide disc is proposed. Load-torque is predicted by soft measure method in the model and its disturbance is canceled by the feed-forward control algorithm. As for the error caused by soft sensing method, inferential control algorithm is applied to avoid it. The compound control algorithm system works to realize the final speed control of guide disc, and its accuracy, robustness and fast response have been proved validated in simulation.
\end{abstract}

Keywords: Guide disk, Load-torque, Soft sense, Feedforward and inferential control

\section{Introduction}

The production of piercing billet is the first manufacturing process of the seamless tubes forming, which makes the solid billet into tube hollow with the cross piercer [1]. The cross piercer with the guide disc has already been applied in production of the seamless tubes widely. In the process of piercing, the guide disc puts axial forward pull on the billet, adds axial acceleration of the tube hollow, makes the elasticity smaller and makes for the process of piercing and the improvement of the quality of the tube hollow [2]. Therefore, it is of great significance to properly control the guide disc rotational speed in order to improve quality of seamless tubes [3].

In this paper, the mathematic model of the guide disc rotational speed system was formed on the base of analyzing working principle and characteristic of the guide disc, the load torque in model was predicted in the way of soft sensing, according to characteristic of disturbance of the strong load torque in the system model and disturbance of the load torque in the system was eliminated in the way of feed-forward control algorithm. Because there are some errors in the model of soft sensing, its inaccurate parts were eliminated in the way of inference control algorithm which had a good control effect for given unmeasurable disturbance, compound controller on the base of feedforward control algorithm and inference control algorithm was designed, which could control the guide disc rotational speed at a expectation when the cross piercer was working, according to the simulation study.

\section{Model of the guide disc rotational speed control system}

Between the hydraulic pump and the hydraulic motor, there is an electric operator. The displacement of the hydraulic motor is changed by regulating opening value of electric operator to regulate guide disc rotational speed. Also, hydraulic guide disc rotational speed is obtained to realize the closed-loop control of the system by installing inductive screw at the place of the hydraulic motor and detecting hydraulic motor rotational speed with the proximity switch. In this paper, the hydraulic system which drove guide disc to move was analyzed and the model of the control system of the guide disc rotational speed was forme $t$.

\subsection{Composition of the hydraulic system}

Most conventional servo-pump variable operating mechanisms adopt hydraulic servo-drive, and its displacement can be changed by the servo valve which controls a hydraulic cylinder to drive the stroking mechanism of the pump. The working oil of the stroking mechanism is supplied by the system directly or by a small pump which is coaxial to variable pump. This is a mature way technically and has a high performance of response, but structure complexity appended by hydraulic actuator and leading-in accurate servo component both increase possibility of trouble [4]. Baosteel adopt a measure of setting extendedly EM (Electromotive Actuator) at the duct pilot of the primary pump of the guide disc hydraulic pump aggregate and regulating the output flow of the primary pump in a way of motor drive to regulate guide disc rotational speed indirectly. EM actuating 
mechanism substitute for original amplifier, proportional valve and hydraulic cylinder. Figure 1 shows the principle of work.

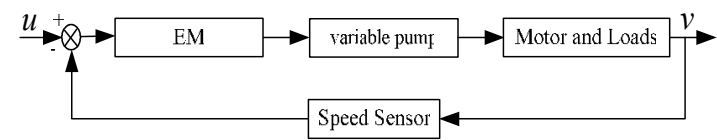

Fig. 1: Guide Disc Rotational Speed System Block Diagram

\subsection{EM actuating mechanism and variable pump}

For conventional variable pump, swashplate drive mechanism is hydraulic ram which makes straight-line reciprocating motion and drive swashplate oscillate to change displacement. In this system, we adopt a measure of setting extendedly EM (Electromotive Actuator) at the duct pilot of the primary pump of the guide disc hydraulic pump aggregate substituting for conventional servo valve and adopt servomotor-ball screw scheme. The running of electromotor will drive ball screw to make straight-line motion then drive plunger pump swashplate to oscillate in order to regulate variation in displacement of pump. EM (Electromotive Actuator) itself is a complex system, but its response speed is much greater than response speed of the hydraulic motor. To simplify system model, in this paper, it will be approximated as a proportional component and its transfer function is as follow:

$$
\frac{z(s)}{U(s)}=k_{z}
$$

In this formula, $z$ is lead-screw displacement, $U$ is input voltage.

For the plunger pump A4VSG500EM2128 in this system, its rotational speed and displacement is stepless adjustable. When the lead-screw displacement of actuating mechanism is $z$, its corresponding swashplate slope angle is $\gamma$

$$
z=L \sin \gamma
$$

In this formula, $L$ is the distance between force point of lead-screw and swashplate hinged point, $\gamma$ is the swashplate slope angle of variable pump. For the change of $\gamma$ is small, it could be considered approximately as:

$$
\gamma(s)=\frac{1}{L} z(s)=K_{\theta} z(s)
$$

\subsection{Motor and loads system}

For there is an accurate gear drive between hydraulic motor and the loads, its drive ratio is $2.1745: 1$, and mechanical rigidity between them is great, to make study easy, in this paper, the hydraulic motor and the loads are considered as one component approximately. For the simplification and the analysis, before analyzing motor-load system model, supposing that the connect conduit between the hydraulic pump and the hydraulic motor is too short to could ignore pressure loss in the conduit and the conduit dynamic, and also supposing that two conduits are just the same, the capacity of both the hydraulic pump and the hydraulic motor are constants; the leaking of both the hydraulic pump and the hydraulic motor are laminar current, the shell pressure of both the pimp and the motor are atmospheric pressure; ignore the outer leaking of the low pressure cavity, the pressure in every cavity is homogeneous and equal, the density and the temperature of the fluid flow are both constants; when outer loads are in a given area, the rotational speed of pump is stationary, the pump displacement is proportional to angle of rotation; ignore nonlinear factor such as friction moment.

According to suppose mentioned above, following flow equation is given:

$$
\begin{gathered}
Q_{p}=D_{p} \omega_{p}-C_{i p}\left(P_{L}-P_{r}\right)-C_{e p} P_{L} \\
D_{p}=K_{p} \gamma_{p} \\
Q_{m}=C_{i m}\left(P_{L}-P_{r}\right)+C_{e m} P_{L}+D_{m} \frac{d \theta_{m}}{d t}+\frac{V_{o}}{\beta_{e}} \cdot \frac{d P_{L}}{d t} \\
Q_{p}=Q_{m}
\end{gathered}
$$

Moment balance equation on motor axis:

$$
D_{m}\left(P_{L}-P_{r}\right)=J_{m} \frac{d^{2} \theta_{m}}{d t^{2}}+B_{m} \frac{d \theta_{m}}{d t}+G \theta_{m}+T_{L}
$$

Others:

$$
\begin{gathered}
\dot{\theta}(s)=s \theta(s) \\
\dot{\theta}(s)=\frac{v(s)}{r}
\end{gathered}
$$

In the formula, $Q_{p}$ is output flow of the pump, $D_{p}$ is radian displacement of the pump, $K_{p}$ is displacement gradient of the pump, $\omega_{p}$ is rotational speed of the pump, $C_{i p}$ and $C_{e p}$ are respectively inner, outer leaking coefficient of the pump, $P$ is return pressure, $P_{L}$ is load pressure, $Q_{m}$ is input flow of the pump, $Q_{m}$ is radian displacement of the motor, $\theta_{m}$ is shaft angle, $C_{i m}$ and $C_{e m}$ are respectively inner, outer leaking coefficient of the motor, $V_{o}$ is total measure of a conduit, including a working cavity of the pump and the motor, the connect conduit and conterminous non- 
working capacity, $\beta_{e}$ is effective volume elastic modulus of the system, $J_{m}$ is total processional moment of the hydraulic motor and the loads(convert to motor axis), $B_{m}$ is total viscous damping coefficient of the hydraulic motor and the loads(convert to motor axis), $G$ is load tensional spring rigidity, $T_{L}$ is outer loads moment acting on the motor axis.

For the simplification and the analysis, many factors such as the inertia, the viscosity, the elasticity, the outer loads, the liquid compressibility and the leaking etc will be ignored, simplify above formula and take its Laplace transform:

$$
v(s)=\frac{\frac{K_{q p}}{D_{m}} r \gamma(s)-\frac{C_{t} r}{D_{m}^{2}}\left(1+\frac{V_{o}}{\beta_{e} C_{t}} s\right) T_{L}(s)}{\frac{s^{2}}{\omega_{h}^{2}}+\frac{2 \zeta_{h}}{\omega_{h}} s+1}
$$

In the formula, $C_{t}$ is the sum of inner vouter leaking coefficient of both the hydraulic pump and the hydraulic motor, $\omega_{h}$ is hydraulic natural frequency, $\zeta_{h}$ is hydraulic dumping, $\omega_{h}$ is $\sqrt{\beta_{e} D_{m}^{2} / V_{o} J_{m}}, \quad \zeta_{h}$ is $\frac{C_{t}}{2 D_{m}} \sqrt{\frac{\beta_{e} J_{m}}{V_{o}}}+\frac{B_{m}}{2 D_{m}^{2}} \sqrt{\frac{V_{o}}{\beta_{e} J_{m}}}$

We can get the transfer function of the shaft angle of hydraulic motor to the tilt angle of variable pump:

$$
\frac{v(s)}{\gamma(s)}=\frac{\frac{K_{q p^{r}}}{D_{m}}}{\frac{s^{2}}{\omega_{h}^{2}}+\frac{2 \zeta_{h}}{\omega_{h}} s+1}
$$

\subsection{Speed sensor}

The speed sensor obtains the signals, converts the signals to the voltage signals and transmits the signals as feedback to input end in which the signals are processed, its dynamic response is considered as a proportional component, its transform function is:

$$
K_{f}=\frac{U(s)}{v(s)}
$$

\subsection{System dynamic block diagram}

According to the equations above, we can get system mathematic model block diagram as Figure 2 shows.

The transform functions of both the system controlled device and the disturbance object can be gotten:

$$
\begin{gathered}
\frac{v(s)}{u(s)}=\frac{K}{\frac{s^{2}}{\omega_{h}^{2}}+\frac{2 \delta_{h}}{\omega_{h}} s+1+K_{1}} \\
\frac{v(s)}{T_{L}(s)}=-\frac{\frac{C_{t} K_{q p^{r}}{ }^{2}}{D_{m}^{3}}\left(\frac{V_{o}}{4 \beta_{e} C_{t}} s+1\right)}{\frac{s^{2}}{\omega_{h}^{2}}+\frac{2 \delta_{h}}{\omega_{h}} s+1+K_{1}}
\end{gathered}
$$

where $K=\frac{K_{z} K_{\theta} K_{q p^{r}}}{D_{m}}, \quad K_{1}=\frac{K_{z} K_{\theta} K_{q p} K_{f}^{r}}{D_{m}}$

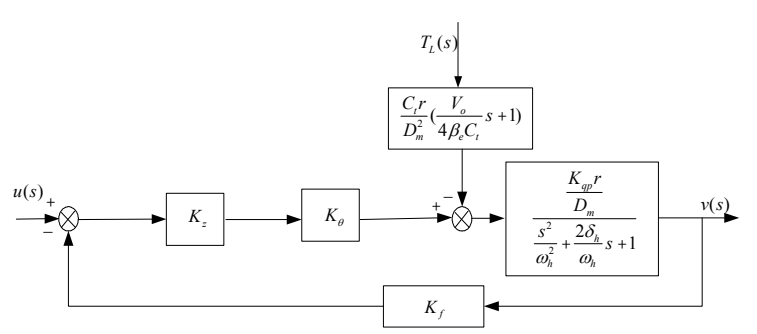

Fig. 2: Motor operator schematic diagram

\section{Soft sensing model of load moment}

According to the models above, it is noted that if we want to control the guide disc rotational speed, the value of guide disc load moment $T_{L}$ must be known, but, in fact, the factors which affect load moment are complex, it is hard to calculate accurately by mechanism formula. In this paper, its measuring problem is solved in a method of PCR soft sensing, because for linearly dependent production data, the PCR method can eliminate its noise interference effectively and has a good regression effect and a high accuracy.

The choice of auxiliary variable is very important in the process of creating soft sensing model. According to production technology and situation of field, we select actual value of both upper and lower roll rotational speed, motor current of both upper and lower roll, location of pusher, physical location of thrust dolly, EMF (motor induced electromotive force) of both upper and lower roll, screw-down actual value of both upper and lower roll, inlet temperature of both left and right guide disc, outlet side temperature of both left and right guide disc etc. fourteen auxiliary variables as input variables of the soft sensing model. These fourteen auxiliary variables affect load moment directly and are easy to obtain. Figure 3 show block diagram of the load moment soft sensing model. The material methods of modeling make reference to literature [5]. 


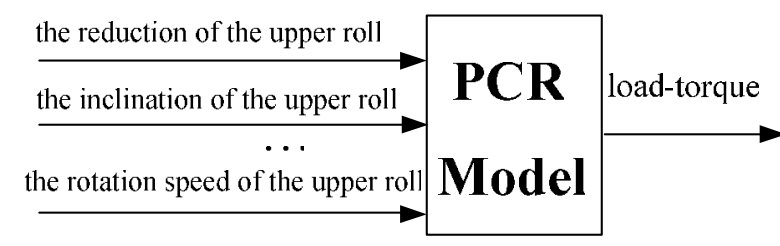

Fig. 3: Soft sensing model schematic diagram

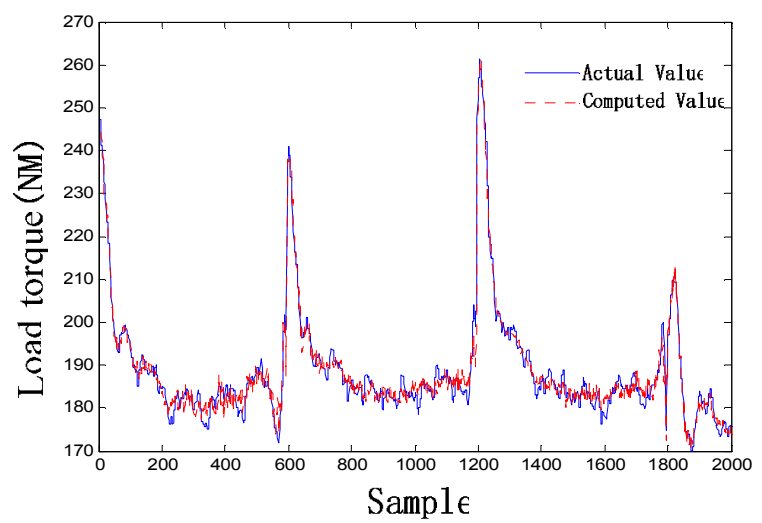

Fig. 4: Check data comparing diagram

Figure 4 shows comparing result of 1000 volumes of check data output and actual output. The solid line is actual data, and the broken line is output data calculated by model. Average error of check data is $2.3 \%$, which could satisfy need of field.

\section{Design of guide disc speed system controller}

\subsection{Principle and characteristics of guide disc speed control system}

Guide disc speed control system is a typical electrohydraulic speed control system with the characteristics of high load rigidity and high accuracy which is common in a hydraulic system. Guide disc speed control system consists of controller, EM actuator, hydraulic pumps, hydraulic motors, guide disc, etc. By calculation, the controller changes the anticipant input signals into control value, and then the EM actuator adjusts the swash plate swing angle of piston pump according to this value, as a result, the displacement of hydraulic pump is changed and hydraulic motor rotational speed is also changed correspondingly, then goals of controlling and adjusting the guide disc speed are reached.

Diesel guide disc has several requirements to the controller:

(1) Higher sensitivity. As the steel tube piercing process is batch production process, and the piercing time used for a tube is only about 12 seconds, therefore the system should be more sensitive to the change of disturbance.

(2) Better quickness for speed regulation. The system should deliver the goods quickly in the case of speed changing.

(3) The regulator should ensure that adjustment highly reliable, simply operation.

(4) Better robustness. That is to say, the antiparameters time-varying ability is better.

\subsection{Design of guide disc speed inferential controller}

The error between the estimate value and real value of the load torque soft-sensing model makes it difficult to get the expectant effect in the control process. In order to solve this problem, inferential control is adopted. Inferential control was originally used to solve the problem caused by non-measurable main output and disturbance, the basic method was later widely used in the process with measurable output and nonmeasurable disturbance, then the inferential control under the condition of measurable output is formed[6][8]. Under the condition that output is measurable and disturbance is immeasurable, the block diagram of inferential control system can be simplified as figure 5 .

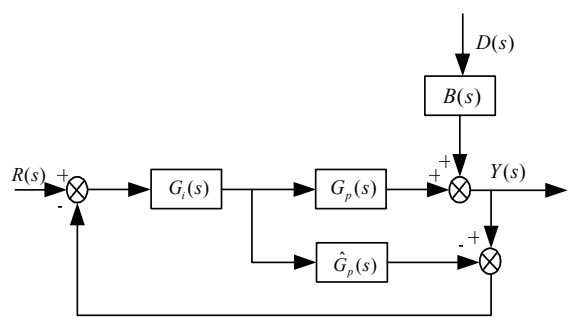

Fig. 5: Inferential control under the condition of measurable output

As shown in figure $5, G_{i}(s)$ is inferential controller, $B(s)$ is disturbance channel transfer function, $G_{p}(s)$ is process channel transfer function, $\hat{G}_{p}(s)$ is process channel estimation model. From Figure 4, the system output is calculated as below:

$$
Y(s)=\frac{\frac{G_{i}(s) G_{p}(s)}{1-G_{i}(s) \hat{G}_{p}(s)}}{1+\frac{G_{i}(s) G_{p}(s)}{1-G_{i}(s) \hat{G}_{p}(s)}} R(s)+\frac{B(s)}{1+\frac{G_{i}(s) G_{p}(s)}{1-G_{i}(s) \hat{G}_{p}(s)}} D(s)
$$

Theoretically, the inferential controller should be $G_{i}(s)=1 / \hat{G}_{p}(s)$, but this type of structure can't be realized in a general way. Therefore, a series-wounded filter $G_{f}(s)$ is linked to the controller. Then 


$$
G_{i}(s)=G_{f}(s) / \hat{G}_{p}(s)
$$

If $\hat{G}_{p}(s)$ follows the form given below:

$$
\hat{G}_{p}(s)=\hat{G}_{p+}(s) \hat{G}_{p^{-}}(s) e^{-\tau s}
$$

where $\hat{G}_{p}(s)$ is composed of all the zero points which lie in the right S-plane. Then the filter can be designed as below:

$$
\hat{G}_{f}(s)=\frac{\hat{G}_{p+}(s) e^{-\tau s}}{\hat{G}_{p+}(0)\left(T_{f} s+1\right)^{n}}
$$

where $\hat{G}_{p+}(0)$ is the static gain of $\hat{G}_{p+}(s), n$ is the rank difference between the denominator polynomial and the numerator polynomial of $\mathrm{s}, T_{f}$ is the filter time constant. After considering the filter, the actual inferential controller is given as below:

$$
G_{i}(s)=\frac{1}{\hat{G}_{p+}(0) \hat{G}_{p-}(s)\left(T_{f} s+1\right)^{n}}
$$

If all models are precise, that is to say $\hat{G}_{p}(s)=G_{p}(s)$, then:

$$
Y(s)=G_{f}(s) R(s)+\left[1-G_{f}(s)\right] B(s) D(s)
$$

It can be seen from the formula above that in actual inferential control system, even if all the models are precise, the main output can not realize fully dynamic tracking under the set point step disturbance and complete dynamic compensation under immeasurable disturbance. The dynamic response of the output $Y(s)$ lies on the filter characteristics. The filter static gain in formula (19) is 1, thus the steady state error of system output is 0 under the action of set point step disturbance and immeasurable disturbance. The system still has a very good steady-state performance[9].

When $\hat{G}_{p}(s) \neq G_{p}(s)$, the system output is

$$
Y(s)=\frac{R(s) G_{p}(s) G_{f}(s)}{\hat{G}_{p}(s)-G_{f}(s)\left(\hat{G}_{p}(s)-G_{p}(s)\right)}+\frac{B(s) D(s) \hat{G}_{p}(s)\left(1-G_{f}(s)\right)}{\hat{G}_{p}(s)-G_{f}(s)\left(\hat{G}_{p}(s)-G_{p}(s)\right)}
$$

Because the static gain of the filter $G_{f}(0)=1$, the steady state error of system output is still 0 under the action of set point step disturbance and immeasurable disturbance.

Inferential control system is an open loop control system mostly drove by specifically immeasurable disturbance, but it doesn't consider other possible disturbances. More over, even for the specifically immeasurable disturbance, if there are model static gain errors, main system output will also have steady state error inevitably. Feedback should be introduced to form inferential-feedback control system in order to eliminate the main output steady state deviation, as shown in Figure 6.

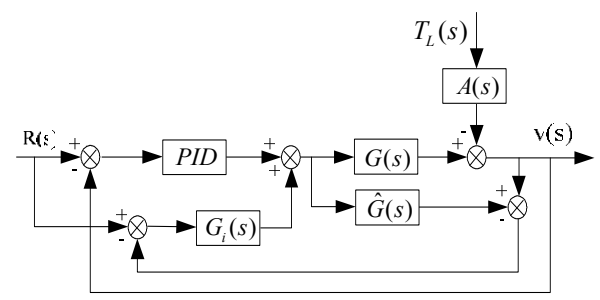

Fig. 6: Inferential-feedback control system

\subsection{Feedforward and inferential combined guide disc speed compound control}

According to the aforementioned characteristics of guide disc speed control system, it's easier to see that this system demands strictly in Steady-state accuracy and response speed. And there is a strong load disturbance in system, so good disturbance rejection ability and good set point tracking ability is required for this control system. Based on this condition, load torque is used for disturbance input in feedforward compensation control[10]. Feedforward control requires that the system mathematical model can be obtained accurately and will not change in the course of operation. Then in the ability of disturbance rejection, the changing of model parameter will result in inaccurate compensation. The stability of inferential control is free of influence caused by model error, but the error will affect the dynamic response of the system. Based on the benefits of the two control methods, feedforward-inferential compound control method is put forward in this paper. This method can not only guarantee the system stability but also have a very good transient response. The system control block diagram is shown in Figure 7.

Under the action of set point, this system can guarantee the static tracing ability and main system output is steady unbiased. In the absence of feedforward control disturbance, the system output can be expressed as:

$$
v(s)=\frac{A(s)\left[G_{f}(s)-1\right]}{1+G(s) P I D} T_{L}(s)
$$

When considering the feedforward control disturbance effect, that is to say, $T_{L}(s)=\hat{T}_{L}(s)+\zeta(s)$, where $\zeta(s)$ is white noise, system output is as follows:

$$
v(s)=\frac{[A(s)-G(s) B(s)]\left[G_{f}(s)-1\right]}{1+G(s) P I D} \hat{T}_{L}(s)+\frac{A(s)\left[G_{f}(s)-1\right]}{1+G(s) P I D} \zeta(s)
$$

where $B(s)={ }^{A(s)} / G(s)$. It's easy to seen that the estimate value of load torque is counteracted by feedforward control.

By comparing formula (23) and (24), it shows 
that due to $\zeta(s)<T_{L}(s)$, the transient response of feedforward-inferential compound control system is better than that of inferential control system.

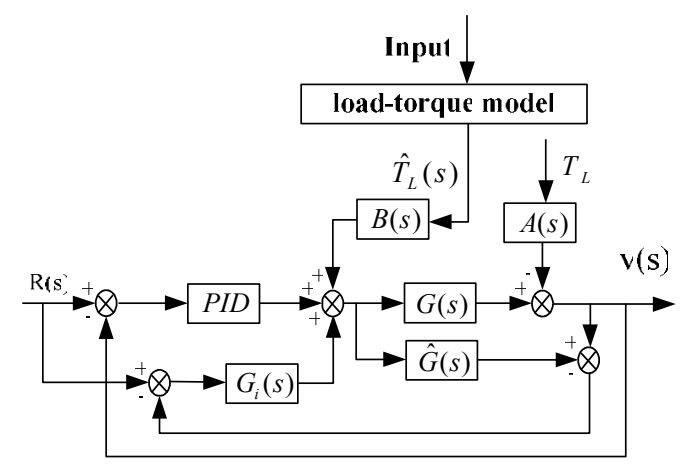

Fig. 7: The block diagram of feedforward-inferential compound control

\section{Simulation analysis}

Using the piercing production field data of Baosteel Pipe Company, a simulation analysis is given to the above conclusion and guide disc speed simulation model is established. The transfer function is $G(s)=\frac{241.3}{s^{2}+5.35 s+600}$, the disturbance model transfer function is $A(s)=\frac{0.3 s+0.18}{s^{2}+5.35 s+600}$, the feedforward controller transfer function is $B(s)=\frac{0.3 s+0.18}{241.3}$, the estimation model of inferential controller $\hat{G}(s)=G(s)$. The inferential controller model is $G_{i}(s)=\frac{s^{2}+5.35 s+600}{60.335 s^{2}+241.3 s+241.3}$, the filter model of inferential controller is $G_{f}(s)=\frac{1}{(0.5 s+1)^{2}}$ The PID parameter of feedforward controller is $K_{p}=10.27, K_{i}=16.98, K_{d}=2.19$ respectively. The PID parameter of inferential controller is $K_{p}=12.64$, $K_{i}=15.4, \quad K_{d}=1.9$ respectively. The PID parameter of compound controller is $K_{p}=20.67, \quad K_{i}=16.9$, $K_{d}=2.1$ respectively. By comparing the inferential algorithm, inferential control algorithm and feedforward-inferential compound control algorithm, the system simulation curves are shown in Figure 8.

Let the guide disc speed be $26 \mathrm{r} / \mathrm{m}$, and add a load torque soft-sensing value when system reaches steady state, meanwhile, a random disturbance with amplitude of 10 and a response time of 0.3 seconds is also added to this system as a system disturbance. As shown in Figure 8, among three control methods, the curve under feedforward control has a slow response speed and a relatively big overshoot, and it requires long time to reach steady state. Curve under inferential control has a bigger overshoot, but regulating time used to reach the steady state is shorter than that of feedforward algorithm, furthermore, it can obtains better control performance combined with PID control. Feedforward-inferential compound control integrates both the benefits so it traces input well and the overshoot is relatively small. This method obtains better control effect. Add disturbance to the system 15 seconds later after the system reaches steady state. Results indicate that the transient response of inferential control is worse than that of the other methods, and the transient response of feedforwardinferential compound control algorithm is quicker than that of feedforward algorithm.

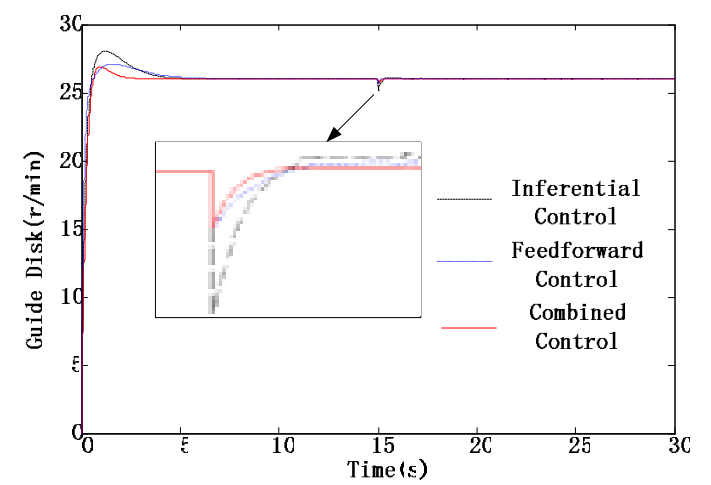

Fig. 8: Response curves of three different control methods

When the transfer function parameter of controlled object changes, takes $G(s)=\frac{200}{s^{2}+5 s+500}$ as an example, the response curve is shown in Figure 9. When the disturbance channel transfer function parameter of controlled object changes, takes $A(s)=\frac{0.2 s+0.1}{s^{2}+5 s+500}$ as an example, the response curve is shown in Figure 10.

From Figure 9 and Figure 10, it's easy to draw a conclusion that feedforward-inferential compound control algorithm, which combines the benefits of both feedforward control algorithm and inferential control algorithm, has very good self-tuning and self-adapting ability. It can control the disc speed to steady state quickly even if the system model parameter is inaccurate. System based on feedforward algorithm or inferential algorithm can't adapt the changes of controlled object fleetly, so there are problems such as longer regulating time and bigger overshoot, etc. 


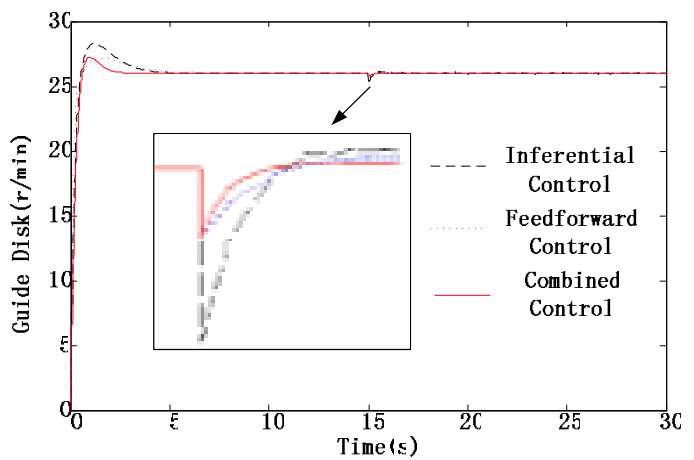

Fig. 9: Response curves before and after the parameter changes

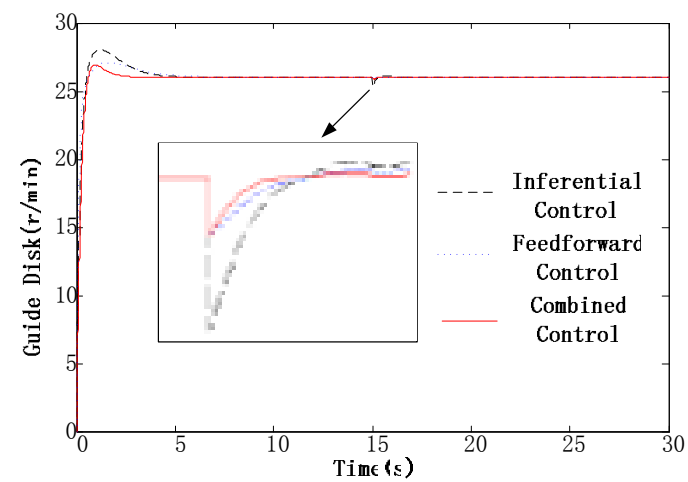

Fig. 10: Response curves before and after the disturbance channel model parameter changes

\section{Conclusions}

This paper takes guide disc as research subject and builds guide disc speed control system model. For the main disturbance in the system-load moment which is difficult to measure directly, PCR is adopted to build soft-sensing model. Finally a compound control method that combines feedforward and inferential control algorithm is adapted in the guide disc speed online real-time control system. By comparing feedforward control algorithm, inferential control algorithm, and feedforward-inferential compound control algorithm, the simulation results shows that feedforward-inferential compound control system requires low sensitivity to the model, with good selfadapting capacity, good robustness, better dynamic characteristics, faster system response speed and better stability.

\section{Acknowledgement}

This work is partially supported by National Nature Science Foundation of China (Grant No. 60674063).

\section{References}

[1] H.J. Pehle, The actuality and development of the seamless tube. Foreign steel tube, 32:47-52, 2003.

[2] Y.H. Shuang, G.Z. Li, Theory of cross process and numerical simulation of production process for steel tubes. Metallurgical Industry Press of China, pp.188-208, 2001.

[3] S. Urbanski, J. Kazanecki, Assessment of the strain distribution in the rotary piercing process by the finite element method. Journal of Mater Process Technol, 45:335-340, 1994.

[4] C.G. Lu, J.L. Li, Analysis and design of the hydraulic control system. Beijing: Coal Industry Press of China, pp.251-259, 1991.

[5] D. Xiao, Z.Z Mao, etc, A modeling method for time series in complex industrial system. The 6th International Conference on Machine Learning and Cybernetics, pp.3423-3427, 2006.

[6] M. Karimi, A. Jahanmiri, M. Azarmi, Inferential cascade control of multi-effect falling-film evaporator. Food Control, 18:1036-1042, 2007.

[7] Z. Xu, T.Y. Cai, W. Wang, A review of inferential control. Information and Control, 27:206-214, 1998.

[8] N. Padhiyar, A. Gupta, Nonlinear inferential multi-rate control of Kappa number at multiple locations in a continuous pulp digester. Journal of Process Control, 16:1037-1053, 2006.

[9] B. Joseph., C. Brosilow, Iinferential control of processes. AIChE J, 24:485-492, 1978.

[10] A.V. Tran, C.J. Chae, R.S. Tucker, Feed-forward control of SOA gain for power equalization in optical burst switching networks. Optics Communications, 266:500-504, 2006. 